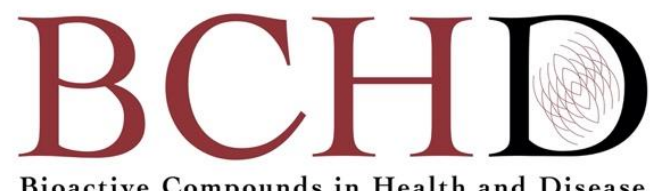

\title{
Ultrafine Bubble Water Usefulness in Fecal Microbiota Transplantation: Recognition of Transplanted Microbiota in Intestinal Epithelial Cells
}

\author{
Shin Shimizu ${ }^{1,2,3 *}$, Katsuaki Dan ${ }^{4}$, Chihiro Tanaka ${ }^{1,2,3}$, Mikiko Tanaka ${ }^{1,2,3}$, Yoshimu Tanaka ${ }^{1,5}$, \\ Masahiko Shirotani $^{1,6}$, Kunihiro Kitamura ${ }^{1,7}$, Kensho Yorozu ${ }^{1,8}$, Masayuki Oehorumu ${ }^{1,9}$, Goro \\ Tsukamoto $^{1,2}$
}

${ }^{1}$ Intestinal Microbiota Transplantation Clinical Study Group, Osaka ; ${ }^{2}$ Symbiosis Inc., Osaka ; ${ }^{3}$ Intestinal Microbiota Transplantation Clinical Research Inc., Osaka; ${ }^{4}$ Division of Research and Development, Research Organization of Biological Activity, Tokyo; ${ }^{5}$ Tanaka Clinic, Osaka ; ${ }^{6}$ Luke's ashiya Clinic, Hyogo; ${ }^{7}$ Kitamura Clinic, Fukuoka; ${ }^{8}$ Yorozu Clinic, Tottori; ${ }^{9}$ LIFE Clinic Tateshina, Nagano

Corresponding author: Shin Shimizu, Intestinal Microbiota Transplantation Clinical Study Group, Osaka, Japan.

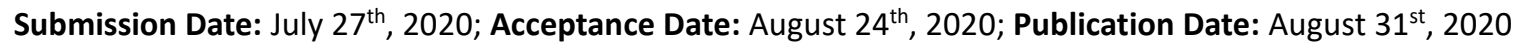

Please cite this article as: Shimizu S., Dan K., Tanaka C., Tanaka M., Tanaka Y., Shirotani M., Kitamura K., et al. Ultrafine bubble water usefulness in fecal microbiota transplantation recognition of transplanted microbiota in intestinal epithelial cells. 2020; 3(8): 141-153 DOI: https://www.doi.org/10.31989/bchd.v3i8.735

\footnotetext{
ABSTRACT

Background: Indications for various diseases such as diabetes mellitus and metabolic syndrome, etc. of Fecal Microbiota Transplantation (FMT) have been investigated. For the establishment of the FMT therapy, the examination of the solvent has not been carried out, though the use of the physiological saline is fixed, at present. We have produced the Mr. Shimizu made ultrafine bubble water (UFB) that produces a larger number of bubbles than existing UFB.

Objective: To verify the usefulness of UFB, we prepared a conventional Saline and UFB-prepared microbial preparation (Bio3); (Bio3/Saline) and (Bio3/UFB). The bacterial preparations, Bio3, contain glycated bacteria, lactic acid bacteria, and butyric acid bacteria. FMT was carried out to the diabetic model mouse using these microbial preparations, and whether the disease state was improved was examined. Cultured intestinal epithelial cells (CaCO-2) were also used to test for differentiation by UFB and Saline from variations in several receptors that recognize microbiota-mRNA. In addition, glucose uptake into cells was measured.
} 
Methods: FMT with (Bio3/UFB) and (Bio3/Saline) was performed in streptozotocin-induced diabetic mice (STZmice) in duplicate at 0,7 days. Blood glucose levels $(7,14$ days) and blood insulin levels (14 days) were measured. Cultured intestinal epithelial cells were also used to test for differentiation by UFB and Saline from change in several receptors that recognize microbiota-mRNA (Toll-like receptors, NOD-like receptors, and RIG-I-like receptors). In addition, glucose uptake into cells was measured using fluorescently labeled glucose analog (NBDG).

Results: UFB could reduce the blood glucose level of STZ-induced diabetic model mice. However, no such effects were observed in Saline. Stimulation of serially diluted Bio3 with UFB-suspension were showed significant alteration in TLR4 and IL-1 $\beta-\mu$ PNA. The amount of glucose uptake in the (Bio3/UFB) group was significantly different at $30 \mathrm{~min}$, inhibited or delayed.

Conclusion: It is concluded that UFB-mediated cross-talk between intestinal bacteria and intestinal epithelial cells and inhibition or delay of intestinal epithelial glucose uptake may have been associated with the reduction of blood glucose levels in diabetic model mice. The superiority of UFB as a suspension used for the transfer of bacteria has been suggested.

\section{INTRODUCTION}

It has become clear that disturbed intestinal microbiota (dysbiosis) is involved not only in digestive diseases such as inflammatory bowel disease and irritable bowel syndrome, but also in various diseases such as metabolic diseases such as diabetes mellitus and metabolic syndrome, autoimmune diseases such as rheumatoid arthritis and multiple sclerosis, and psychiatric disorders such as autism and depression [1, 2]. Fecal Microbiota Transplantation (FMT) has attracted attention as a bacteriological treatment with fewer side effects in an attempt to improve this dysbiosis.

FMT was shown to have a significantly higher recurrence-free cure rate for recurrent Clostridium difficile infection (CDI) in 1958 [3], and in 2013 compared with the conventional treatment, oral vancomycin [4]. Currently, the Infectious Diseases Society of America (IDSA) guideline strongly recommends FMT for recurrent CDI [5]. Ulcerative colitis (UC): New protocols for UC have been investigated, and combinatorial therapy (Antibiotics-FMT: A-FMT) with allergic antimicrobial therapy (Amoxicillin, Fosfomycin, Metronidazole: AFM) and FMT has also been advocated [6, 7].

For the establishment of the FMT therapy, there is a room for examination such as administration route, dosage, administration frequency, selection method of the donor, etc.. Especially, on the preparation of the bacterial solution, the examination of the solvent, etc. has not been carried out, though the use of the physiological saline is fixed, at present.

Ultrafine bubble water is a technology that generates foam of $1 \mu \mu$ or less into the liquid. Because of the fine volume of the foam, it continues to exist in the liquid for a long period of time, and its application such as cleaning ability and preservation of food is spreading from the potential characteristics of the foam surface. We have produced the Mr. Shimizu made ultrafine bubble water (UFB; International Public Number: WO2019/168034 A1 and trade mark registration number: 2020-57176) that produces a larger number of bubbles than existing UFB, and have obtained some evidence assuming that the bacteria can be drawn into the epithelial lining of the intestinal mucosa by using it as a solvent for the 
implanted bacterial suspension (in preparation for posting).

In the present study, we investigated the changes in blood glucose levels and the blood insulin levels at the end of treatment in streptozotocin-induced diabetic mice (STZ-mice) after enema with conventional Saline and UFB-prepared microbial preparation (Bio3) [8]; hereinafter referred to as (Bio3/Saline) and (Bio3/UFB), respectively. The bacterial preparations, Bio3, used here contain glycated bacteria, lactic acid bacteria, and butyric acid bacteria.

Enterobacteria are foreign to the organism but are not eliminated by the intestinal immune system and are symbiotic. Much remains unknown as to how intestinal epithelial cells recognize and accept and eliminate enterobacteria transplanted from vitro. Toll-like receptors (TLRs $[9,10]$, Nod-like receptors (NLRs) [11,12], and RIG-I like receptor (RLRs) [13], are known as receptors for intestinal epithelial cells that recognize mycotic components (Figure 1.). Among TLRs, TLR2 recognizes Gram-positive bacteria, and TLR4 recognizes Gram-negative bacteria. The presence of Nod1, Nod2, NLRP3, NLRC4, etc. has been confirmed in NLRs, and mRNA levels of IL-1? , known as the main responses including mRNA level and NLRP3, NLRC4 of Nod1, Nod2, and RIG-I, MDA5, LGP2-mRNA as RLRs were analyzed by quantitative RT-PCR. Differentiation between UFBs and Saline was investigated from variations in mRNA levels recognizing transplanted microbial products (Bio3).

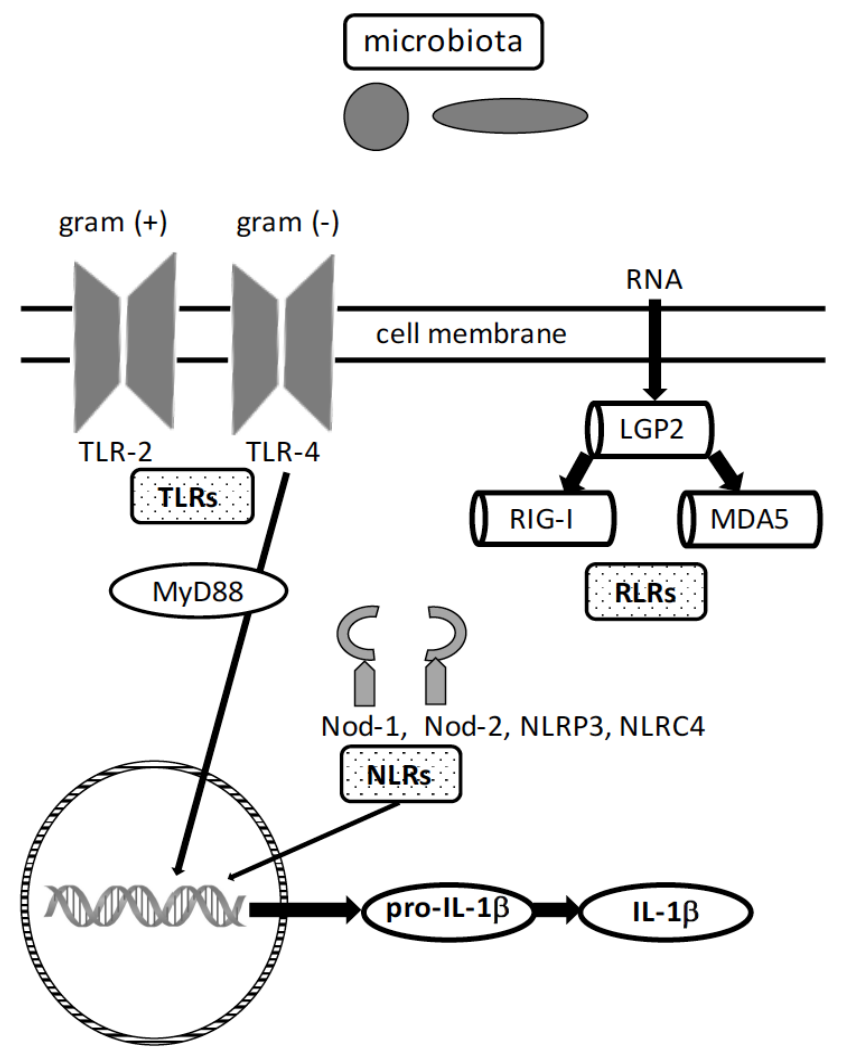

Figure 1: Receptors in intestinal epithelial cells that recognize bacteria.

The following were examined: Toll-like receptors (NLRs ; Nod-1, Nod-2, NLRP3, NLRC4) which existed in the plasma membrane, Nod-like receptors (NLRs ; Nod-1, Nod-2, NLRP3, NLRC4) which existed in the cytoplasm, and RIG-I like receptor (RLRs; RIG-I, MDA5, LGP2). Among TLRs, TLR2 recognizes Gram-positive bacteria and TLR4 recognizes Gramnegative bacteria. mRNA levels of IL-1b, known as major responses of NLRs, were also examined. 
In addition, it is assumed that the uptake of glucose is inhibited or delayed in intestinal epithelial cells, etc., when the disease state improvement effect is observed in STZ mouse in which the insulin production function is almost destroyed.

Therefore, the uptake of glucose was also examined when (Bio3/UFB) and (Bio3/Saline) were added to cultured intestinal epithelial cells.

A part of the usefulness of UFB as a preparation solvent of the microbiota was recognized under the condition of diabetes mellitus disease state model mouse and cultured intestine epithelial cell.

\section{MATERIALS \& METHODS}

Materials: UFB was granted by the Intestinal Flora Transplantation Study Group. Saline purchased the Japanese Physiological Saline (NISSIN PHARMACEUTICAL) solution. Bacterial formulations (Bio Three ${ }^{\circledR}$; manufactured by Takeda Consumer Healthcare Co., Ltd.) were donated by the Intestinal Flora Transplantation Clinical Study Group. Glucose CIl-test Wako (FUJIFILM Wako Pure Chemical Corporation) was used to measure blood glucose levels. Ultra Sensitive Mouse Insulin ELISA Kit (Morinaga Institute of Biological Science, Inc.) was used to measure blood insulin levels. 2-NBDG(2-N-(7Nitrobenz-2-oxa-1,3-diazol-4-yl)Amino)-2-Deoxyglucose) (NBDG) was purchased from ThermoFisher (Carlsbad, CA). Mouse insulin, streptozotocin (STZ), and other molecular biological reagents were purchased from FUJIFILM Wako Pure Chemical Corporation and used.

Animals: CD-1 (ICR) mice (male, 4 weeks old) purchased from Japanese Charles River Inc. were previously housed for 1 week and then used for experiments. Experiments were conducted in accordance with the Animal Experiment Guideline of the Biological Activity Research Organization. Cell culture: Intestinal epithelial cells
(CaCO-2): RCB0988 were imported from European Collection of Cell Cultures (ECACC) via a distributor (K.A.C.) and cultured in a $5 \% \mathrm{CO} 2,37^{\circ} \mathrm{C}$ incubator by designated dedicated medium (MEM + 20\% FBS + 0. $1 \mathrm{mM}$ NEAA; non-essential amino acids) for use.

\section{Preparation of model animals for diabetes mellitus and} FMT experiment: $100 \mathrm{mmol} / \mathrm{L}$ citrate buffer (pH 4.5) STZ was administered to mice at $40 \mathrm{mg} / \mathrm{kg}$, iv. One week later, blood was drawn from the mouse ocular venous plexus using a capillary, and the mice were divided into four groups of six mice each so that each group would be equal from the measured blood glucose levels:

1) Intact control group (Control), 2) UFB-prepared microbial solution group (Bio3/UFB), 3) Saline-prepared microbial solution group (Bio3/Saline), and 4) Insulin treatment group (In.T). The bacterial fluid was injected using a single-dose needle from the anus to the large intestine with $1 \mathrm{ml}$ per mouse on the first day of the experiment and twice on the seventh day. Insulins were given daily $1 \mathrm{IU} / 0.1 \mathrm{ml}$ ip to mice 14 times up to 1-14 days, at fixed times of 6 PM. Blood glucose levels were collected twice on days 7 and 14 using a capillary from the mouse ophthalmic venous plexus, and blood insulin levels were measured in the jugular vein under ether anesthesia on day 14 , respectively. The experimental protocol is shown in Figure 2.

\section{Recognition of Bacterial Products in Intestinal Epithelial}

Cells: CaCO-2 at a concentration of 50000 cells $/ \mathrm{ml}$ and $2 \mathrm{ml}$ (a total of 0.1 million cells) were pre-cultured in 35 $\mathrm{mm}$-dish for 48 hours before being used for the experiment. After removing the old culture solution in dish and washing the cell surface with phosphate buffered saline (PBS), $2 \mathrm{ml}$ of a culture solution made of UFB in which Bio3 powder was added at various concentrations (10-fold serial dilutions from $1 \%$ to 
$0.0001 \%)$ was added, and culture was continued. The same operation was carried out on the culture solution made of Saline.

Total RNA of cells was extracted from each dish at each elapsed time $(1,4$, and $8 \mathrm{~h})$.

Total RNA extract: Trizol ${ }^{\circledR}$ Reagent (ThermoFisher Scientific Co., Ltd.) was used, and the work was carried out according to the protocol. After removing the culture solution of the Petri dish and washing off the cell surface with PBS, transfer the pipetted solution to a $1.5 \mathrm{ml}$ test tube after adding $1 \mathrm{ml}$ of Trizol. Chloroform, $0.2 \mathrm{ml}$, is added thereto, stirred, and then centrifuged. (The centrifugal conditions are all $4^{\circ} \mathrm{C}$ and $12000 \mathrm{rpm}, 15 \mathrm{~min}$ below.) After centrifugation, transfer the upper layer section to a new $1.5-\mathrm{ml}$ test tube, add $0.5 \mathrm{ml}$ of isopropyl alcohol to it, stir, and then centrifuge. After removing the upper layer, add $1.0 \mathrm{ml}$ of ethanol, mix by inversion, and then centrifuge. The upper layer was removed, and 50? of ultrapure water (RNase free) was added to the precipitate completely volatilized with ethanol to serve as a Total RNA sample. A portion of this sample was used to quantify RNAs levels by $(1 \mu \lambda)$, NanoDrop (ThermoFisher Scientific).

* CD-1 (ICR) mice (male, 4 weeks old) purchased from Japanese Charles River Inc. were previously housed for 1 week.

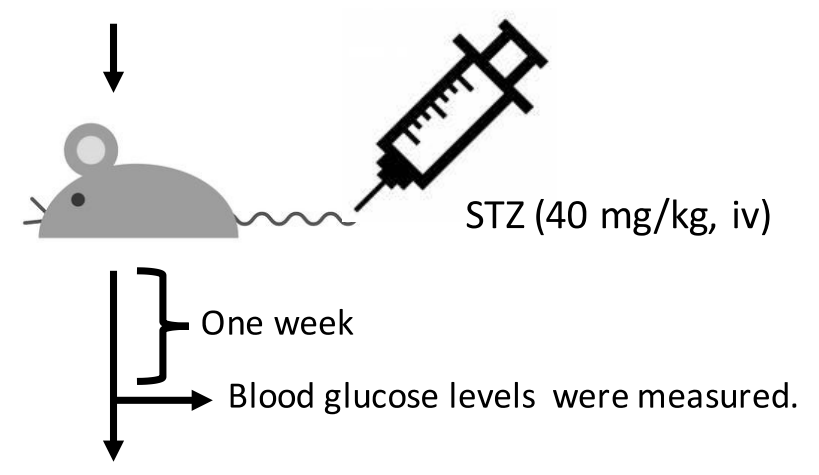

* The mice were divided into four groups of six mice each so that each group would be equal from the measured blood glucose levels:
1)Intact control group (Control),
2) UFB-prepared microbial solution group (Bio3/UFB),
3) Saline-prepared microbial solution group (Bio3/Saline),
4) Insulin treatment group (In.T).

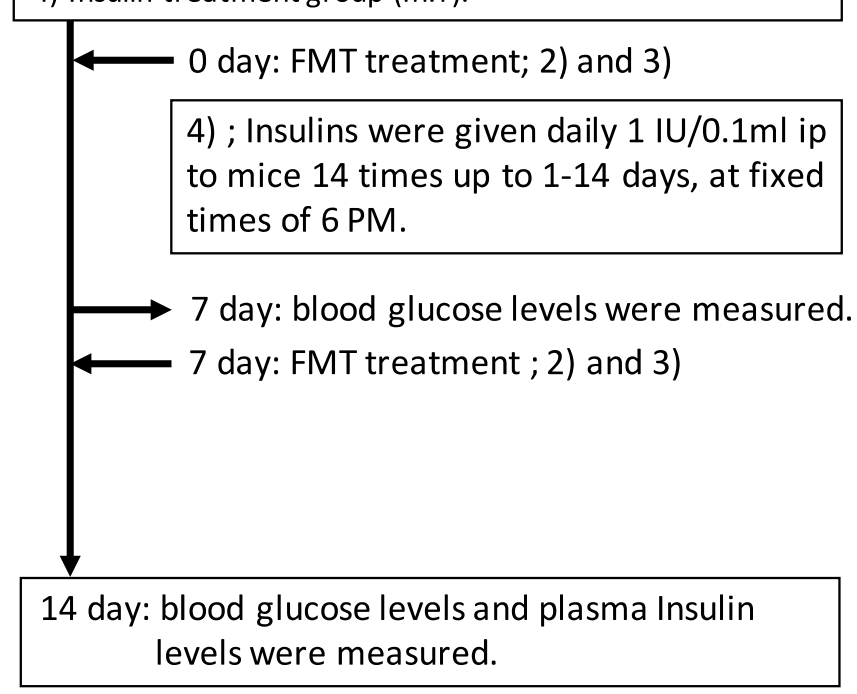

Figure 2. Experimental design. 
Quantitative RT-PCR (RT-qPCR): Expression levels of receptor-related genes (TLRs [14], NLRs [15, 16], and RLRs [17]), which recognize bacterial components, and glyceraldehyde-3-phosphate dehydrogenase (GAPDH as Internal control [14] were measured by RT-qPCR.

Total RNA, $500 \mathrm{ng}$ was used as a Template for PCR, and cDNA synthesis by reverse-transcription reactions (Reverse Transcriptase; RT reactions) and a one-step RTPCR in which quantitative PCR could be performed in one tube were performed using Luna Universal One-Step RTqPCR Kit. The PCR device was made of Takara and Thermal Cycler Dice Real Time System II. As one system,
Luna Universal One-Step Reaction Mix (2x), 10 ??l, Luna WarmStart RT Enzyme Mix (20x), 1 ?l, Forward primer (10 ?M), $0.8 \mu \lambda$, Reverse primer, $0.8 \mu \lambda$, Template RNA, variable were used, and Nuclease-free Water was adjusted to 20 回. The reaction was carried out at $95^{\circ} \mathrm{C}$ for $30 \mathrm{~s}$ for $1 \mathrm{cycle}$, and at $95^{\circ} \mathrm{C}$ for $5 \mathrm{~s}$ and $60^{\circ} \mathrm{C}$ for $30 \mathrm{~s}$ for 50 cycles. For RT-qPCR, primers for various targets and housekeeping genes (GAPDH) were used (Table 1.). Those that fluctuated more than 4-fold as the expression of mRNA (two cycles as PCR-reaction) were judged as significant differences.

Table 1. Primers for PCR

\begin{tabular}{|c|c|c|c|}
\hline Gene & Primer & & References \\
\hline \multirow[t]{2}{*}{ TLR2 } & Forward; & 5'-TTA TCC AGC ACA CGA ATA CAC AG-3' & 14 \\
\hline & Reverse; & 5'-AGG CAT CTG GTA GAG TCA TCA A-3' & \\
\hline \multirow[t]{2}{*}{ TLR4 } & Forward; & 5'-AGA CCT GTC CCT GAA CCC TAT-3' & 14 \\
\hline & Reverse; & 5'-CGA TGG ACT TCT AAA CCA GCC A-3' & \\
\hline \multirow[t]{2}{*}{ Nod1 } & Forward; & 5'-TCC AAA GCC AAA CAG AAA CTC-3' & 15 \\
\hline & Reverse; & 5'-CAG CAT CCA GAT GAA CGT G-3' & \\
\hline \multirow[t]{2}{*}{ Nod2 } & Forward; & 5'-GAA GTA CAT CCG CAC CGA G-3' & 15 \\
\hline & Reverse; & 5'-GAC ACC ATC CAT GAG AAG ACA G-3' & \\
\hline \multirow[t]{2}{*}{ IL-1b } & Forward; & 5'-GCT GCT TCC AAA CCT TTG AC-3' & 16 \\
\hline & Reverse; & 5'-AGC TTC TCC ACA GCC ACA AT-3' & \\
\hline \multirow[t]{2}{*}{ RIG-I } & Forward; & 5'-AGG AAA ACT GGC CCA AAA CT-3' & 17 \\
\hline & Reverse; & 5'-TTT CCC CTT TTG TCC TTG TG-3' & \\
\hline \multirow[t]{2}{*}{ MDA-5 } & Forward; & 5'-CTG CTG CAG AAA ACA ATG GA-3' & 17 \\
\hline & Reverse; & 5'-TGC CCA TGT TGC TGT TAT GT-3' & \\
\hline \multirow[t]{2}{*}{ LGP-2 } & Forward; & 5'-ATG TGA ACC CCA ACT TCT CG-3' & 17 \\
\hline & Reverse; & 5'-CAA TGC TGC AGG AAG TCA AA-3' & \\
\hline \multirow[t]{2}{*}{ GAPDH } & Forward; & 5'-AGG GCT GCT TTT AAC TCT GGT-3' & 14 \\
\hline & Reverse; & 5'-CCC CAC TTG ATT TTG GAG GGA-3' & \\
\hline
\end{tabular}

Glucose uptake in bacterial formulation-supplemented intestinal epithelial cells (CaCO-2): Experimental methods were performed by using a Mojika $L$ and other methods [18]. To $35 \mathrm{~mm}$-dish and 96-well plate of CaCO$2\left(5 \times 10^{4}\right.$ cells/ well), $100 \mu \mathrm{M}$ NBDG, which is a fluorescent labeling glucose analog, was added to glucose (-) culture solution for 4 hours, and then washed at intervals of elapsed time $(0,30,60,120,180 \mathrm{~min})$. The amount of labeled fluorescence (Ex; $485 \mathrm{~nm}, \mathrm{Em} ; 535 \mathrm{~nm}$ ) incorporated into the cells was measured with a fluorescent plate reader (SYNERGY HT micro plate reader: Bio Tek, Tokyo, Japan). In part, observations were made 
under fluorescent microscopy to acquire fluorescent images (BZ-X710, Keyence, Osaka, Japan) and joined its software BZ-analyzer (Keyence). Experiments were performed under identical conditions with $n=4$, and results are presented as mean values \pm SD (standard deviation) for each group. Significant differences were tested by One way ANOVA.

\section{RESULTS}

FMT-effects of (Bio3/UFB) on diabetic model-mice: Mice that became hyperglycemic by STZ administration had their blood glucose levels measured before starting FMT. The mean blood glucose levels (mg/dl) in the four groups

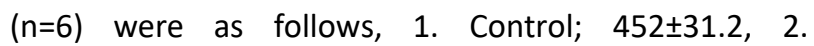

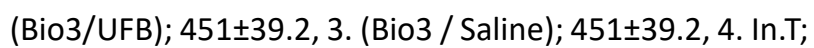

$456.3 \pm 57.1$, and the experiment was started so that the difference between each group was minimal. Changes in blood glucose levels after FMTs were performed in diabetic mice as a ratio to untreated controls (Figure 3a). Significant reduction in blood glucose was observed by FMT (0, 7 days) with (Bio3/UFB) a reduction of approximately $80 \%$ compared to a reduction of up to $60 \%$ in Positive control insulin-treated group, and (Bio3/Saline) no difference was observed in the FMT group compared to untreated controls. Blood insulin levels at the end of the study were close to the detection limit in both groups, but the level in the (Bio3/UFB) group tended to increase but was not as high as that in the insulin-treated group. (Bio3/Saline) values were almost similar to those of intact controls in the group (Figure $3 \mathrm{~b}$ ).

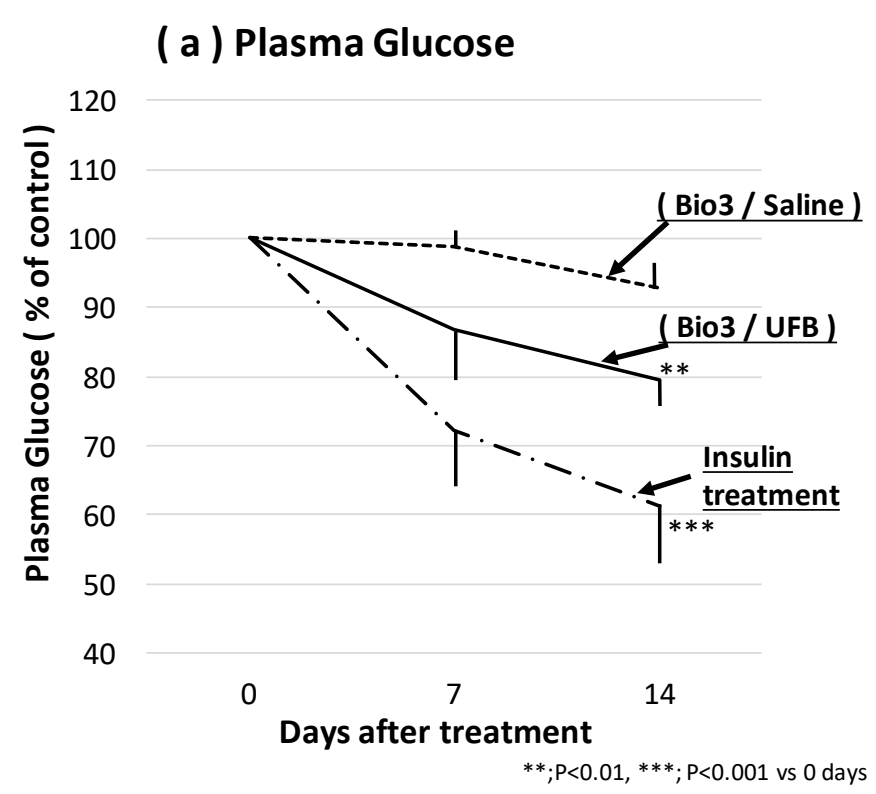

(b) Plasma Insulin

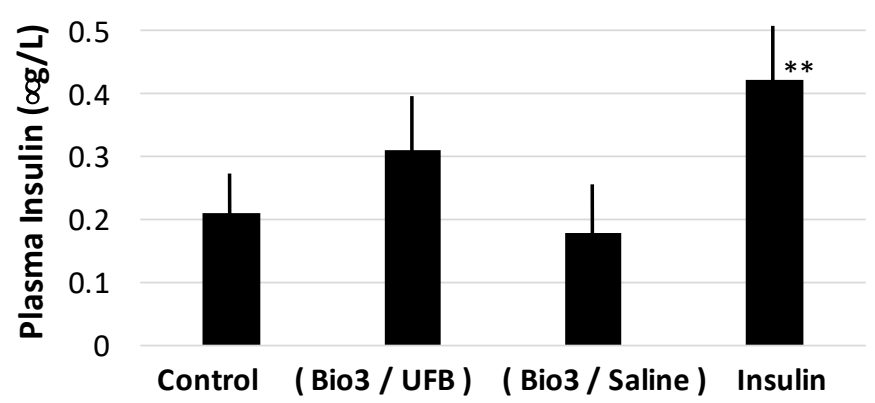

**; $\mathrm{P}<0.01$ vs Control

Figure 3: The FMT effect of (Bio3/UFB) on diabetic model mice. (a): Ratio of changes in blood glucose levels to intact controls. ${ }^{* * P}<0.01,{ }^{* * *} \mathrm{P}<0.001$ vs 0 day. (b): Blood insulin levels at the end of the experiment. ${ }^{* * P}<0.01$ vs Control. 
Expression of Bacterial Recognition Receptor-mRNA of Intestinal Epithelial Cells in Response to (Bio3/UFB) additions: For changes in bacterial recognition receptormRNA to (Bio3/UFB) addition, the results of examining whether or not mRNA expression levels fluctuate in a concentration-dependent manner at 4 hours after addition are shown as increases or decreases in expression levels prior to stimulation (Figure 4.). In the quantitative RT-PCR, a Fold change greater than or equal to 2-fold for Bio3 free was judged as the significant difference.

Stimulation of serially diluted Bio3 with UFBsuspension resulted in no variation in TLR2-mRNA levels, but a concentration-dependent significant reduction was observed with TLR4. In the other mRNA, both Nod1, Nod2 tended to increase, but the differences were not significant. On the other hand, IL-1? ?-mRNA showed a significant increase in a concentration-dependent manner.

By adding more than $0.1 \%$ of (Bio3/UFB), the recognizing bacterial recognition receptor-mRNA was confirmed to exhibit significant levels of variation. (0.1\%Bio3/UFB) and UFB alone stimulated differences in bacterial recognition receptor-mRNA expression were studied for 1, 4, and 8 hours after treatment (Figure 5ah). The addition of UFB alone did not alter mRNA levels, but the addition of (Bio3/UFB) tended to elevate Nod-1, Nod-2, IL-1? values of 4 hours as a peak.

We investigated whether differences by UFB and Saline occur at the same Bio3 concentrations and stimulus times (Figure 6.). The vertical axis of the graphs shows the expression level of mRNA by (Bio3/Saline) from the expression level of mRNA by (Bio3/UFB) in Delta under the same condition.

For each mRNA, the expression was generally higher at 4 hours after stimulation (Bio3/UFB), and the greatest differences were observed. Although the difference between UFB and Saline was not clear in both TLR-2, TLR4 , it appeared with a large difference in Nod-1, IL-1 $\beta$ of NLRs.
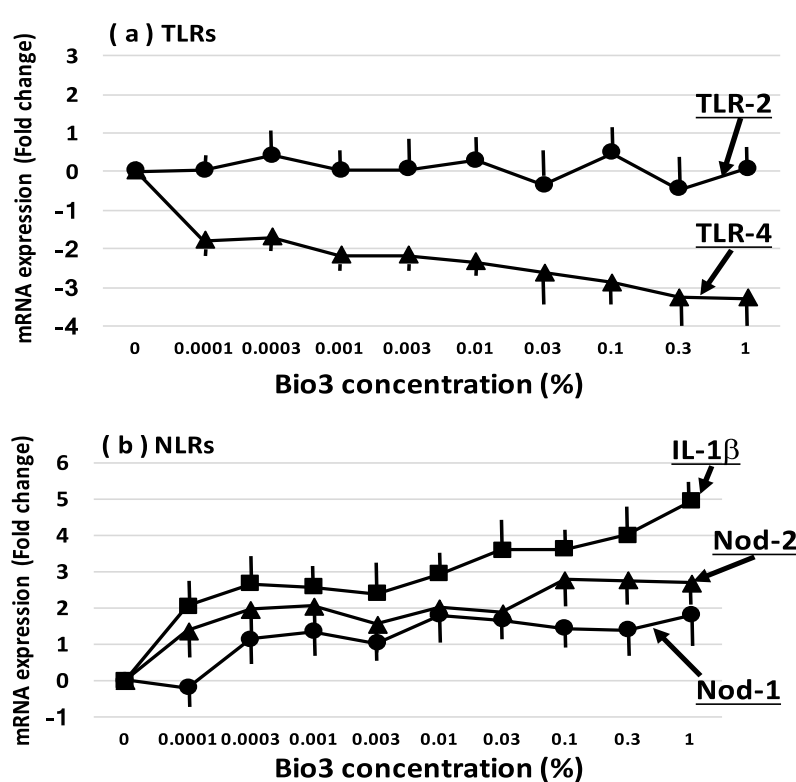

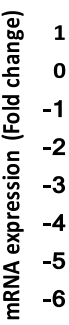

(c) RLRS

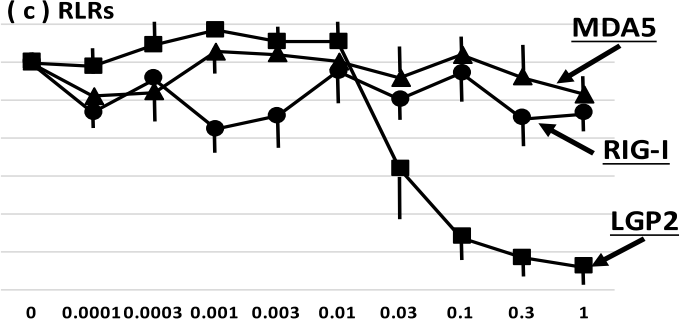

Bio3 concentration (\%)

Figure 4: Expression of bacterial recognition receptor-mRNA of intestinal epithelial cells in response to UFBsupplementation. For the concentration-dependence of Bio3, responses at 4 hour post-stimulation are shown for each receptor group. (a): TLRs, (b): NLRs, (c): RLRs. A Fold change greater than or equal to 2-fold for Bio-3 free was judged as the significant difference. 
(a) TLR-2

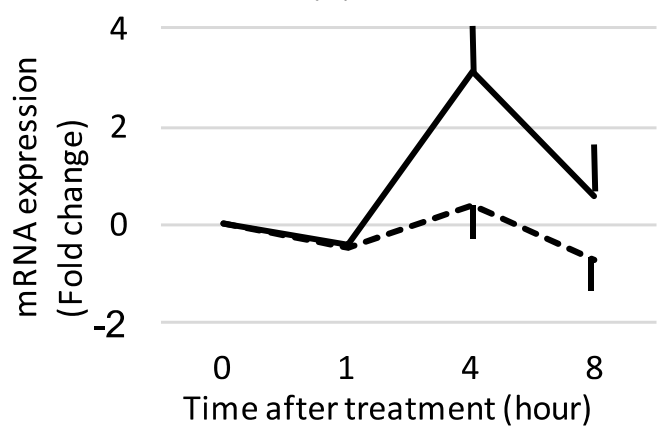

(c) Nod-1

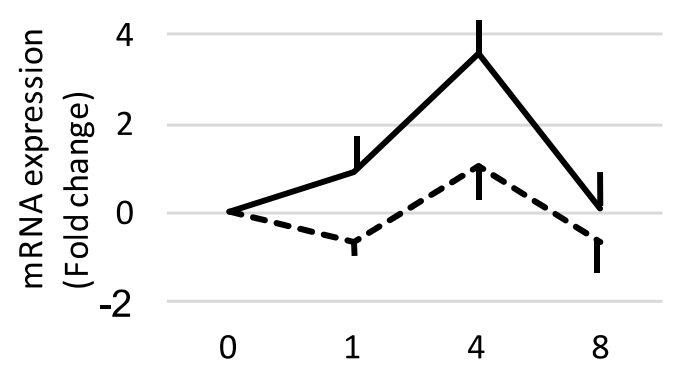

Time after treatment (hour)

(e) IL-1 $\beta$

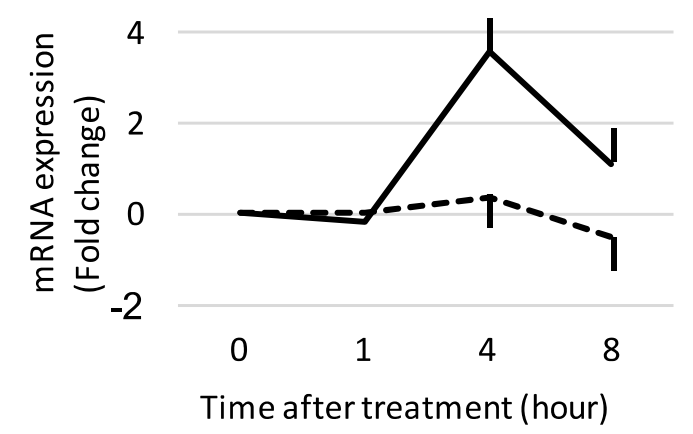

(g) MDA5

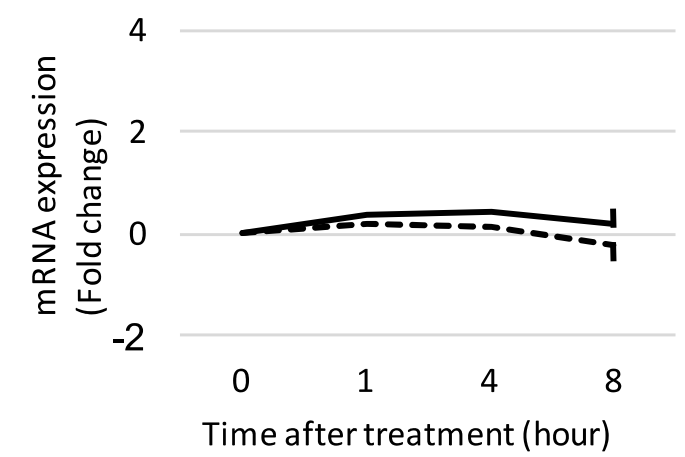

(b) TLR-4

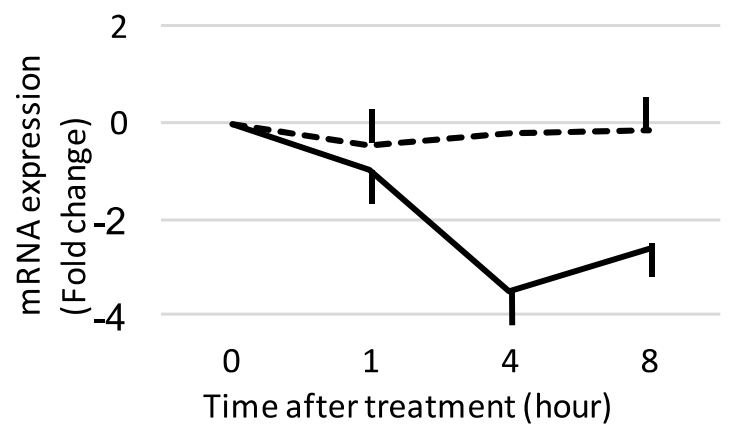

(d) Nod-2

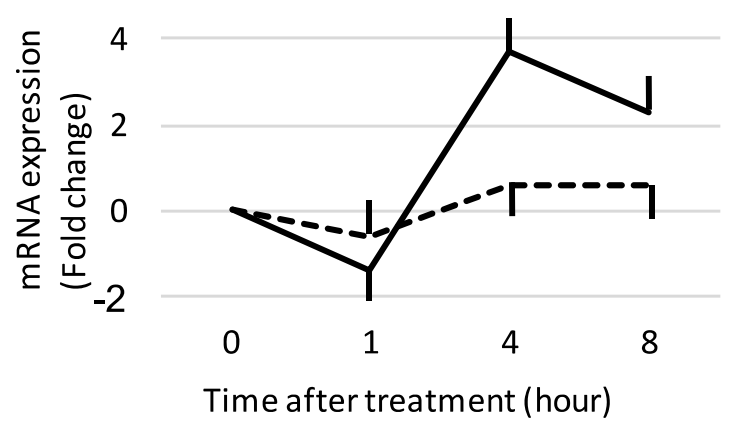

(f) RIG-I

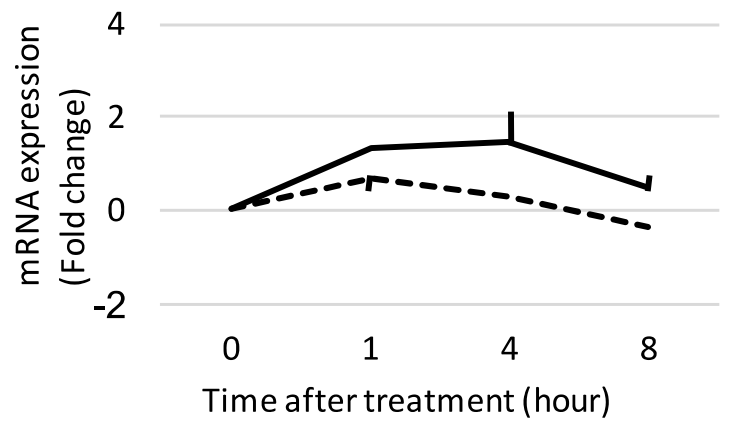

(h) LGP-2

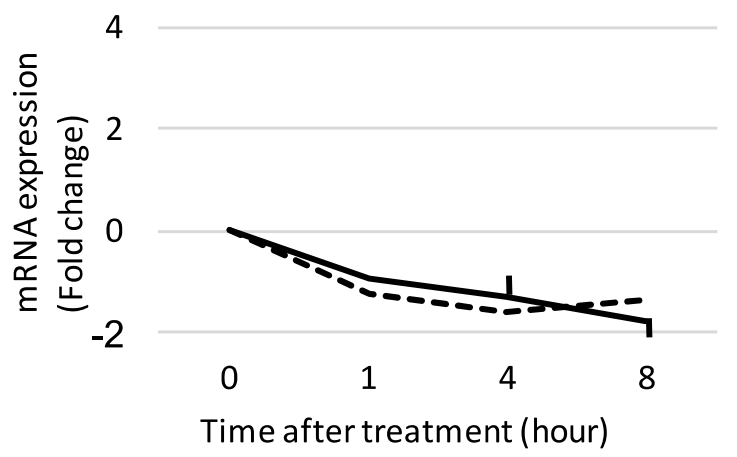

Figure 5: Expression of bacterial recognition receptor-mRNA of intestinal epithelial cells in response to UFBsupplementation. For the concentration-dependence of Bio3, responses at 4 hour post-stimulation are shown for each receptor group. (a): TLRs, (b): NLRs, (c): RLRs. A Fold change greater than or equal to 2-fold for Bio-3 free was judged as the significant difference. 


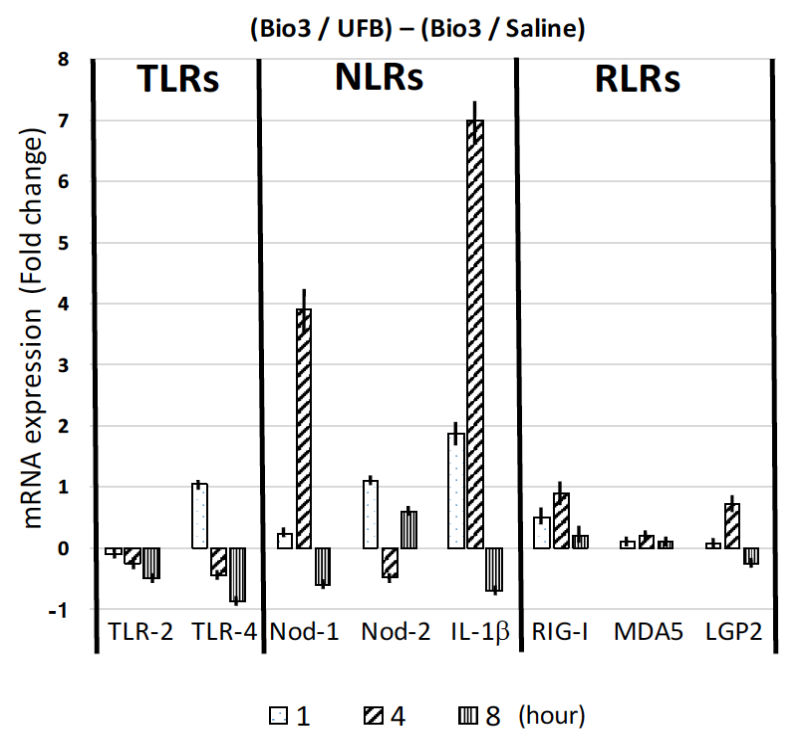

Figure 6: Expression of bacterial recognition receptor-mRNA of intestinal epithelial cells in response to UFBsupplementation. For the concentration-dependence of Bio3, responses at 4 hour post-stimulation are shown for each receptor group. (a): TLRs, (b): NLRs, (c): RLRs. A Fold change greater than or equal to 2 -fold for Bio-3 free was judged as the significant difference.

Effects of UFBs in glucose uptake of intestinal epithelial cells: (Bio3/UFB) and (Bio3/Saline) to investigate whether differences in glucose uptake occur in intestinal epithelial cells stimulated for 4 hours, the amount of intracellular fluorescent at $30 \mathrm{~min}$ after adding NBDG was measured at 5 sites each in Dish (Figure 7.). Fluorescence images showed representative examples. The mean fluorescent intensity at five sites was 17800 Unit in (Bio3/Saline) compared to 14200 Unit in (Bio3/UFB). The amount of fluorescence in the cells was then measured over time, showing that compared with the amount of fluorescence in the (Bio3/Saline) group, the amount of glucose uptake in the (Bio3/UFB) group was significantly different at 30 min, inhibited or delayed (Figure 8.).

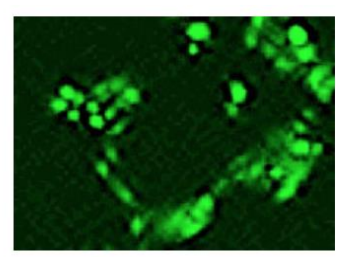

(Bio3 / Saline)

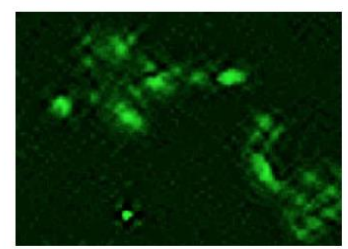

(Bio3 / UFB )

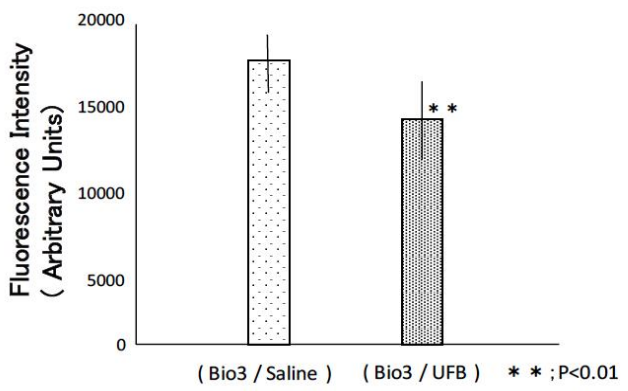

Figure 7: The effects of UFBs in glucose uptake of intestinal epithelial cells. (after NBDG stimulus, 30 min.). Images of intracellular NBDG were shown in the upper panels of Figure 7. The lower panel of Figure 7 shows the quantitative data for intracellular NBDG determined as fluorescence intensity. ${ }^{* *} \mathrm{P}<0.01$ vs (Bio3/Saline). 

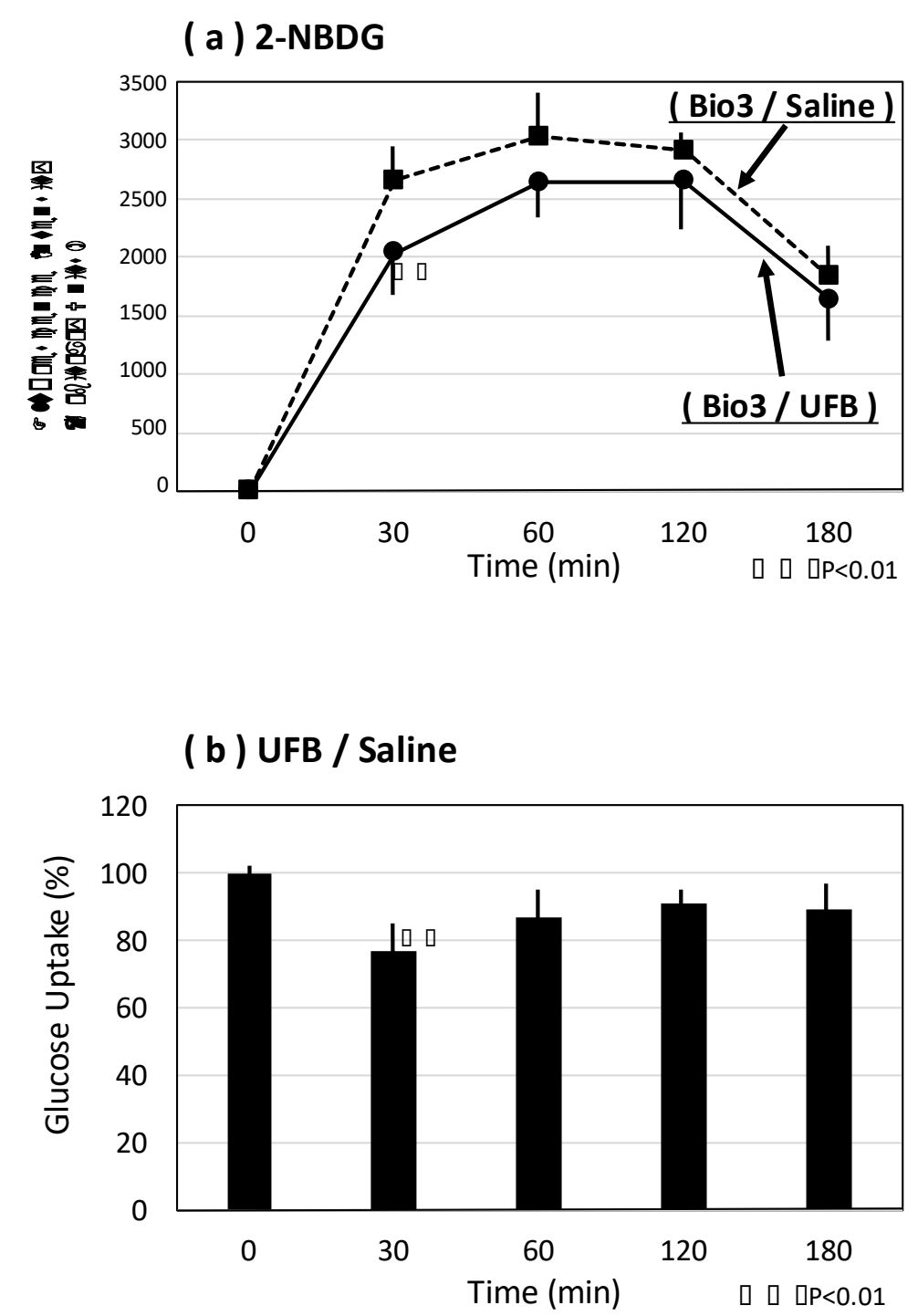

Figure 8: The effects of UFBs in glucose uptake of intestinal epithelial cells. (time course). (a): Mean fluorescent quantities at five locations at each measurement time, (b): ratio of UFBs to Saline in glucose uptake. ${ }^{* * P<0.01}$ vs (Bio3/Saline).

\section{DISCCUSION}

Inspired by the fact that microbiota preparation is poorly investigated during the spread of diseases indicated for FMT, the usefulness of UFBs that we can produce was compared with the conventional Saline. In order to differentiate UFB from Saline, we used a commercialized Bio-3, considering that it is desirable that the bacteria to be administered should be a fixed combination product.

Since FMT is clinically targeted for type 2 diabetes mellitus $[2,19]$, we aimed at a relatively mild pathology with a small single dose of STZ $(40 \mathrm{mg} / \mathrm{kg}$, iv). The persistence of Insulin secretions is evident from Figure $3 b$ results. UFB could reduce the blood glucose level of STZ- induced diabetic model mice. However, no such effects were observed in Saline (Figure 3a). Based on the results, the usefulness of UFB as a solvent for microbiota of FMT is expected. Blood Insulin levels were not significantly elevated, and Insulin secretory stimuli did not explain this hypoglycemia (Figure 3b). In addition, there was a tendency for glucose uptake in intestinal epithelial cells to be suppressed or delayed compared with Saline (Figure 7,8 ). This is also a differentiation from Saline, which may be partly attributed to a reduction in blood glucose levels by inhibiting glucose uptake. GLP-1 secretions may be related to alterations in intestinal microbiota, specifically Lachnobacterium, B. adolescentis 
and Coriobacteriaceae, in patients with type 2 diabetes [20]. In the future, the relevance between FMTs using UFBs and Incretin secretions such as GLP-1 should be investigated.

In a comparative study between (Bio3/UFB) and UFB alone, UFB alone did not alter the receptors in epithelial cells, indicating that cross-talk between microbiota and cells is also required and helpful (Figure $5 \mathrm{a}-\mathrm{h}$ ).

Stimulation of serially diluted Bio3 with UFBsuspension did not alter TLR2-mRNA levels, but significant reduction was observed in TLR4, suggesting that Gram-negative bacteria were recognized.

On the other hand, IL-1?-mRNA showed a significant increase in a concentration-dependent manner.

These results suggest that (Bio3/UFB) stimulates NLRP3 of NLRs and NLRC4 system to recognize the fungus. Little response was seen in RLRs, but these are receptors that recognize mainly RNA molecules and may not operate upon Bio3 stimuli (Figure $5 \mathrm{f}, \mathrm{g}, \mathrm{h}$ ). Usually, when the organism was recognized, the associated mRNA levels were thought to be elevated. There is a possibility that Bio3 used in this study was formulated specifically for three types of bacteria, or that negative feedback was applied because the amount added was large for the cells, but this could not be confirmed.

\section{CONCLUSION}

It is concluded that UFB-mediated cross-talk between intestinal bacteria and intestinal epithelial cells and inhibition or delay of intestinal epithelial glucose uptake may have been associated with the reduction of blood glucose levels in diabetic model mice. Thus, the superiority of UFB as a suspension used for the transfer of bacteria has been suggested.

Abbreviations: FMT : Fecal Microbiota Transplantation; CDI : Clostridium difficile infection; IDSA: Infectious Diseases Society of America; UC: Ulcerative colitis; AFM: Amoxicillin, Fosfomycin, Metronidazole; UFB: ultrafine bubble water; STZ-mice: streptozotocin-induced diabetic mice; TLRs: Toll-like receptors; NLRs: Nod-like receptors; RLRs: RIG-I like receptor; NBDG: 2-NBDG(2-N-(7Nitrobenz-2-oxa-1,3-diazol-4-yl)Amino)-2-

Deoxyglucose); In.T: Insulin treatment group; PBS: phosphate buffered saline; RT-qPCR: Quantitative RTPCR; GAPDH: glyceraldehyde-3-phosphate dehydrogenase; SD: standard deviation

Conflict of interest: The authors declare no conflicts of interest.

Funding: The authors declare no external funding.

Authors' contributions: All authors contributed to this work. Shimizu M, Dan K, Tanaka C, and Tanaka M designed the research. Shimizu M, Dan K, and Tanaka C carried out the experiments and analyzed the results. All authors interpreted the results and designed the research strategy. Shimizu M, and Dan K prepared the manuscript.

\section{REFERENCES}

1. Nemoto H, Kataoka K, Ishikawa H, Ikata K, Arimochi H, Iwasaki T, Ohnishi Y, Kuwahara T, Yasutoma K: Reduced diversity and imbalance of fecal microbiota in patients with ulcerative colitis. Dig Dis Sci 2012, 57:2955-2964.

2. Kostic $A D$, Vavier RJ, Gevers D: The microbiome in inflammatory bowel disease: current status and the future ahead. Gastroenterology 2014, 146:1489-1499.

3. Eiseman B, Silen W, Bascom GS, Kauvar AJ:Fecal enema as an adlunct in the treatment of pseudomembranous enterocolitis. Surgery 1958:44:854-859.

4. van Nood E, Vrieze A, Nieuwdorp $M$, Fuentes $S$, Zoetendal EG, de Vos WM, Visser CE, Kuijper EJ, Bartelsman JF, Tjissen JG, Speelman P, Dijkgraaf MG, Keller JJ:Duodenal infusion of donor feces for recurrent Clostridium difficle. N Engl J Med 2013, 368:407-415. 
5. McDonald LC, Gerding DN, Johnson S, Bakken JS, Carroll KC, Coffin SE, Dubberke ER, Garey KW, Gould CV, Kelly C, Loo V, Shaklee Sammons J, Sandora TJ, Wilcox MH: Clinical Practice Guidelines for Clostridium difficile Infection in Adults and Children: 2017 Update by the Infectious Diseases Society of America (IDSA) and Society for Healthcare Epidemiology of America (SHEA) . Clin Infect Dis. 2018, 19;66(7):e1-e48.

6. Ohkusa T, Kato K, Terao S, Chiba T, Mabe K, Murakami K, Mizokami Y, Sugiyama T, Yanaka A, Takeuchi Y, Yamato S, Yokoyama T, Okayasu I, Watanabe S, Tajiri H, Sato N; Japan UC Antibiotic Therapy Study Group: Newly developed antibiotic combination therapy for ulcerative colitis: a double-blind placebo-controlled multicenter trial. Am J Gastroenterol 2010, 105:18201829.

7. Ishikawa D, Sasaki T, Osada T, Kuwahara-Arai K, Haga K, Shibuya T, Hiramatsu K, Watanabe S: Changes in intestinal microbiota following combination therapy with fecal microbial transplantation and antibiotics for ulcerative colitis. Inflamm Bowel Dis 2017, 23: 116-125.

8. BIO-THREE H Powder TOA BIOPHARMA CO., LTD. [http://www.toabio.co.jp/cms/toa/forlign/item_inde x_e.html]

1. 9. Imler J L, Hoffmann J A: Toll receptors in innate immunity. Trends Cell Biol 2001, 11(7):304-311.

9. Kawai T, Akira S: Toll-like receptors and their crosstalk with other innate receptors in infection and immunity. Immunity 2011, 34(5):637-650.

10. Ting J P-Y, Lovering R C, Alnemri E S, Bertin J, Boss J M, Davis B K, Flavell R A, Girardin S E, Godzik A, Harton J A, Hoffman H M, Hugot J P, Inohara N, Mackenzie A, Maltais L J, Nunez G, Ogura Y, Otten L A, Philpott D, Reed J C, Reith W, Schreiber S, Steimle V, Ward P A: The NLR gene family: a standard nomenclature 2008, 28(3): 285-287.

11. Inohara N, Chamaillard M, McDonald C, Nuñez G:
NOD-LRR proteins: role in host-microbial interactions and inflammatory disease. Annu Rev Biochem 2005, 74:355-383.

12. Yoneyama M, Fujita T: RNA recognition and signal transduction by RIG-I-like receptors. 2009, 227(1): 5465.

13. Badi S A, Khatami S, Irani S, Siadat S D: Induction effects of bacteroides fragilis derived outer membrane vesicles on toll like receptor 2, Toll like receptor 4 genes expression and cytokines concentration in human intestinal epithelial cells. Cell J 2018, 21(1): 57-61.

14. Kim J G, Lee S J, Kagnoff M F: Nod1 is an essential signal transducer in intestinal epithelial cells infected with bacteria that avoid recognition by toll-like receptors. Infect Immun 2004, 72(3): 1487-1495.

15. Xu Y W, Xing R X, Zhang W H, Li L, Wu Y, Hu J, Wang C, Luo Q L, Shen J L, Chen X: Toxoplasma ROP16I/III ameliorated inflammatory bowel diseases via inducing M2 phenotype of macrophages. World J Gastroenterol 2019, 25(45): 6634-6652.

16. Ohta K, Fukui A, Shigeishi H, Ishida Y, Nishi H, Tobiume $\mathrm{K}$, Takechi M, Kamata N: Expression and function of RIG-I in oral keratinocytes and fibroblasts. Cell Physiol Biochem 2014, 34:1556-1565.

17. Mojica L, Luna-Vital D A, Mejia E G: Black bean peptides inhibit glucose uptake in $\mathrm{CaCO}-2$ asenocarcinoma cells by blocking the expression and translocation pathway of glucose transporters. Toxicology Reports 2018, 5:552-560.

18. Kang Y, Cai Y: Gut microbiota and obesity: implications for fecal microbiota transplantation therapy. Hormones 2017: 16(3): 223-234.

19. Cornejo-Pareja I, Martín-Núñez GM, Roca-Rodríguez MM, Cardona F, Coin-Aragüez L, Sánchez-Alcoholado L, Gutiérrez-Repiso C, Muñoz-Garach A, FernándezGarcía JC, Moreno-Indias I, Tinahones FJ: H. pylori Eradication Treatment Alters Gut Microbiota and GLP1 Secretion in Humans. J Clin Med 2019, 8(4): 451-467. 